\title{
The phenotype associated with a large deletion on MECP2
}

\author{
Ami Bebbington ${ }^{1}$, Jenny Downs ${ }^{1,2}$, Alan Percy ${ }^{3}$, Mercé Pineda ${ }^{4}$, Bruria Ben Zeev ${ }^{5}$, Nadia Bahi-Buisson ${ }^{6}$ \\ and Helen Leonard ${ }^{\star 1}$
}

\begin{abstract}
Multiplex ligation-dependent Probe Amplification (MLPA) has become available for the detection of a large deletion on the MECP2 gene allowing genetic confirmation of previously unconfirmed cases of clinical Rett syndrome. This study describes the phenotype of those with a large deletion and compares with those with other pathogenic MECP2 mutations. Individuals were ascertained from the Australian Rett Syndrome and InterRett databases with data sourced from family and clinician questionnaires, and two case studies were constructed from the longitudinal Australian data. Regression and survival analysis were used to compare severity and age of onset of symptoms in those with and without a large deletion. Data were available for 974 individuals including 51 with a large deletion and ages ranged from 1 year 4 months to 49 years (median 9 years). Those with a large deletion were more severely affected than those with other mutation types. Specifically, individuals with large deletions were less likely to have learned to walk (OR $0.42,95 \% \mathrm{Cl}: 0.22-0.79, P=0.007)$ and to be currently walking (OR $0.53,95 \% \mathrm{Cl}: 0.26-1.10, P=0.089$ ), and were at higher odds of being in the most severe category of gross motor function (OR 1.84, 95\% Cl: $0.98-3.48, P=0.057$ ) and epilepsy (OR $2.72,95 \% \mathrm{Cl}: 1.38-5.37, P=0.004$ ). They also developed epilepsy, scoliosis, hand stereotypies and abnormal breathing patterns at an earlier age. We have described the disorder profile associated with a large deletion from the largest sample to date and have found that the phenotype is severe with motor skills particularly affected.
\end{abstract}

European Journal of Human Genetics (2012) 20, 921-927; doi:10.1038/ejhg.2012.34; published online 4 April 2012

Keywords: rett syndrome; large deletion; phenotype

\section{INTRODUCTION}

Rett syndrome is a severe neurodevelopmental disorder, which is clinically diagnosed ${ }^{1}$ and usually associated with a mutation in the MECP2 gene. ${ }^{2}$ The first line of genetic testing involves mutation screening and sequencing of exons 3 and 4 for small sequence changes. $^{3}$ Over 200 MECP2 mutations have been identified ${ }^{4}$ with some point mutations (p.R106W, p.R133C, p.R168X, p.R255X, p.R270X, p.R294X, p.R306C and p.T158M) occurring more commonly. Subsequent to sequencing, multiplex ligation-dependent probe amplification (MLPA) has also became available for the detection of deletions of one or more exons. ${ }^{5}$ Archer et al ${ }^{6}$ found that many of the large deletions had a breakpoint in the deletion prone region of exon 4 and were of variable size. Used as a complementary method to DNA sequencing, MLPA now allows for the identification of a pathogenic $M E C P 2$ mutation in the group of females with a clinical presentation of Rett syndrome in whom a pathogenic MECP2 mutation would not previously have been identified.

Both classic and atypical presentations have been reported in those with large deletions, ${ }^{6-8}$ but these studies have had relatively few cases $(n=12-22)$ or have not included a comparison group of individuals with other types of mutations. Larger studies have confirmed that a large deletion is associated with a severe phenotype, for example, in comparison with the phenotype of cases with the p.R $133 \mathrm{C}^{9}$ or a C-terminal deletion, ${ }^{10}$ which are both considered to be generally mild. Examination of a large sample of individuals with a large deletion in terms of general severity and the specific aspects of phenotype is needed to advance our understanding of this more recently identified mutation group.

Combining data collected as part of InterRett, ${ }^{11}$ an international Rett syndrome phenotype database, with data from the Australian Rett Syndrome Database (ARSD), ${ }^{12}$ a population-based cohort, the phenotype associated with a large deletion is described in detail and compared with the phenotype of cases with other common mutations.

\section{METHODS}

The ARSD was established in 1993 and is a population-based register of Australian RTT cases born since $1976 .{ }^{13}$ Data are collected through the submission of family and clinician questionnaires on recruitment. Additional questionnaire data have been collected every 2 to 3 years since $2000 .{ }^{14}$ InterRett was established in $2003^{15}$ and cases are ascertained through a variety of sources including parent support groups, the listserv Rettnet and also bulk submission of de-identified data provided by clinicians outside Australia from countries such as Spain, France, Israel and Canada. InterRett questionnaires are completed online by families and clinicians at one time point. All cases were verified as Rett syndrome by fulfilling diagnostic criteria ${ }^{16}$ and having a pathogenic

\footnotetext{
${ }^{1}$ Telethon Institute for Child Health Research, Centre for Child Health Research, University of Western Australia, Perth, Western Australia, Australia; ${ }^{2}$ School of Physiotherapy and Curtin Health Innovation Research Institute, Perth, Western Australia, Australia; ${ }^{3}$ Civitan International Research Centre, University of Alabama, Birmingham, AL, USA; ${ }^{4}$ Hospital Sant Joan de Deu, Barcelona, Spain; ${ }^{5}$ Safra Pediatric Hospital, Sheba Medical Center, Ramat-Gan, Israel; ${ }^{6}$ Université René Descartes - Paris V, Hopital Necker Enfants Malades, Paris, France

*Correspondence: Dr H Leonard, Telethon Institute for Child Health Research, Centre for Child Health Research, University of Western Australia, Perth, Western Australia 6872, Australia. Tel: +61 0894897790; Fax: +61 0894897700; E-mail: hleonard@ichr.uwa.edu.au
}

Received 9 June 2011; revised 9 November 2011; accepted 7 December 2011; published online 4 April 2012 
MECP2 mutation. Large deletions were defined in this study as deletions of all or most of an exon of $M E C P 2$, occurring most commonly in our subjects as a deletion of all or a substantial part of exons 3 and 4 .

Data to describe the presence of individual aspects of phenotype including age of onset of common co-morbidities were available in the questionnaire responses. Data for items contained in the Kerr, Percy and Pineda severity scores were coded and summed to calculate severity scores (in which higher scores denote greater severity) as previously described. ${ }^{17}$ Data from the Spanish cohort were used to calculate the Pineda scale scores but not the Kerr or Percy scale scores, because of differences in questionnaire design (and therefore data collection), but were used in analyses on the individual aspects of Rett syndrome severity where this data was collected on the Spanish cohort.

Data held in the longitudinal ARSD were interrogated to describe the early developmental features, the presentation of the diagnostic criteria and the longitudinal course of functional abilities, scoliosis, epilepsy, sleep, behavioural and breathing disorders in two girls with a large deletion and to illustrate the variability in their phenotypes. Data were sourced from physician and family questionnaires completed at ascertainment to the study, ${ }^{13}$ from follow-up family questionnaires ${ }^{14,18}$ and from video data provided in 2004 and 2007. ${ }^{19}$ Case study one is 10 years old; data were available from eight time points. Case study two is 21 years old; data were available from nine time points.

Ethical approval for the study was provided by the ethics committee of Princess Margaret Hospital in Western Australia.

\section{Data analysis}

Missing data in the severity-scale individual items were imputed using Multiple Imputation using Chained Equations, a Stata program. ${ }^{20,21}$ Severity scale and item scores were described with standard descriptive statistics. Binary or ordinal logistic regression was used to assess the influence of a large deletion on individual item scores in all except the 198 Spanish cases. Linear regression was used to compare the presence of a large deletion with all other cases for each severity score. Univariate and multivariate (adjusted for the effects of age group (youngest) and data source (InterRett)) regression models were analysed. Time-to-event (survival) analysis (with the median ages at onset and $P$ values from the log-rank test of equivalence of survival curves) was used to investigate the risk of onset for age of features of Rett syndrome between those with or without a large deletion.

\section{RESULTS}

At the time of data analysis a total of 974 females (746 non-Australian InterRett and 228 from the ARSD) were eligible to be included in this study. They ranged in age from 1 year 4 months to 49 years with a mean age of 11.53 years (median 9 years) at ascertainment or most recent contact where applicable. For all 974 individuals, those with a large deletion had a mean age of 9.14 years (median 7 years) at most recent contact and those with another pathogenic MECP2 mutation had a mean age of 11.66 (median 9.15 years) at most recent contact. Excluding the Spanish cases $(n=776)$, those with a large deletion had a mean age of 9.43 years (median 7 years) at most recent contact and those with another pathogenic MECP2 mutation had a mean age of 12.46 (median 10.00 years) at most recent contact. More detailed genetic information was available for 43 of the 51 individuals with a large deletion and at least 300 base pairs were deleted from exons 3 and 4 in 22 (51.2\%), exon 4 in $19(44.2 \%)$, exon 1 and 2 in $1(2.3 \%)$ and exon 2 and 3 in $1(2.3 \%)$ of cases.

The Kerr severity scores ranged from 7 to 26 points out of a possible 30 , with higher scores denoting more severe abnormality in the common features of Rett syndrome. The average Kerr severity score for the 206 cases with complete information was 17.0 points and imputed severity was similar for the remaining 768 cases using the Stata command MICE. The Percy score ranged from 7 to 36 out of a possible 45 points, with average complete-case severity of 22.4 . The Pineda score severity ranged from 6 to 26 points (maximum possible 31 points) with average complete-case severity of 15.99 points. The frequency distribution by each of the common mutations is shown in Table 1, with the early truncating mutations including those nonsense mutations occurring before amino acid 310, with the exception of the individuals with p.R168X, p.R255X, p.R270X and p.R294X.

Using the three severity scales, individuals with a large deletion had higher levels of overall severity compared with all other MECP2 mutations, having the highest average Pineda and Percy scores, and second highest average Kerr score (Table 2). Those with a large deletion had similar clinical severity to those with a p.R106W, p.R255X, p.R270X, early truncating and p.R168X mutation, but were significantly more severe than those with a p.R133C, p.R294X, p.R306C or C-terminal deletion (Figure 1). The high scores in the specific domains of breathing abnormalities, hand use, epilepsy, language and gross motor function contributed to the high severity scores. The presence of a large deletion was associated with decreased odds of ever having learned to walk (OR $0.42,95 \%$ CI: $0.22-0.79$, $P=0.007$ ) and of currently walking (OR $0.53,95 \%$ CI: $0.26-1.10$, $P=0.089$ ). A small proportion of those with a large deletion currently used words, though the proportions who had ever used babble or words were similar for those with and without large deletions (Figure 2). Generally those with a large deletion were less likely than those with other mutations to have air swallowing or kyphosis reported (Table 3).

Table 1 Frequency distribution for each category of pathogenic mutation for each data source

\begin{tabular}{|c|c|c|c|}
\hline Type of pathogenic mutation & InterRett cases $(n=746)$ & $A R S D$ cases $(\mathrm{n}=228)$ & All cases $(n=974)$ \\
\hline Early truncating ${ }^{a}$ & $37(5 \%)$ & $14(6 \%)$ & $51(5 \%)$ \\
\hline Large deletion & $32(4 \%)$ & $19(8 \%)$ & $51(5 \%)$ \\
\hline p.R168X & $82(11 \%)$ & $26(11 \%)$ & $108(11 \%)$ \\
\hline p.R255X & $94(13 \%)$ & $16(7 \%)$ & $110(11 \%)$ \\
\hline p.R270X & $55(7 \%)$ & $21(9 \%)$ & $76(8 \%)$ \\
\hline p.R294X & $53(7 \%)$ & $20(9 \%)$ & $73(7 \%)$ \\
\hline p.R106W & $30(4 \%)$ & $10(4 \%)$ & $40(4 \%)$ \\
\hline p.R133C & $47(6 \%)$ & $20(9 \%)$ & $67(7 \%)$ \\
\hline p.T158M & $99(13 \%)$ & $23(10 \%)$ & $122(13 \%)$ \\
\hline p.R306C & $59(8 \%)$ & $14(6 \%)$ & $73(7 \%)$ \\
\hline C terminal deletion & $70(9 \%)$ & $24(11 \%)$ & $94(10 \%)$ \\
\hline Other & $88(12 \%)$ & $21(9 \%)$ & $109(11 \%)$ \\
\hline
\end{tabular}

aThe early truncating mutations category includes those with nonsense mutations occurring before amino acid 310, with the exception of the individuals with p.R168X, p.R255X, p.R270X and p.R294X mutations. 
Table 2 Univariate and multivariate relationships calculated with linear regression between clinical severity of individuals with a large deletion in comparison to all other cases for each severity scale

\begin{tabular}{|c|c|c|c|c|c|}
\hline \multirow[b]{2}{*}{ Severity score } & \multirow[b]{2}{*}{ Mutation group } & \multicolumn{2}{|c|}{ Univariate } & \multicolumn{2}{|c|}{ Multivariate relationships ${ }^{\mathrm{a}}$} \\
\hline & & Mean $(95 \% \mathrm{Cl})$ & P-value & Adjusted mean $(95 \%$ Cl) & $\mathrm{P}$-value \\
\hline \multirow[t]{2}{*}{$\operatorname{Kerr}(n=776)$} & No large deletion & $15.76(15.32-16.19)$ & Baseline & $12.92(12.17-13.67)$ & Baseline \\
\hline & Large deletion & $16.69(15.15-18.23)$ & 0.26 & $14.30(12.81-15.78)$ & 0.06 \\
\hline \multirow[t]{2}{*}{ Percy $(n=776)$} & No large deletion & 21.33 (20.70-21.96) & Baseline & 18.77 (17.57-19.97) & Baseline \\
\hline & Large deletion & $25.20(22.85-27.54)$ & 0.002 & $22.81(20.36-25.26)$ & 0.001 \\
\hline \multirow[t]{2}{*}{ Pineda $(n=974)$} & No large deletion & $16.05(15.70-16.40)$ & Baseline & $16.09(15.45-16.72)$ & Baseline \\
\hline & Large deletion & $18.29(16.84-19.75)$ & 0.003 & $18.36(16.80-19.91)$ & 0.003 \\
\hline
\end{tabular}

aadjusting for age and data source

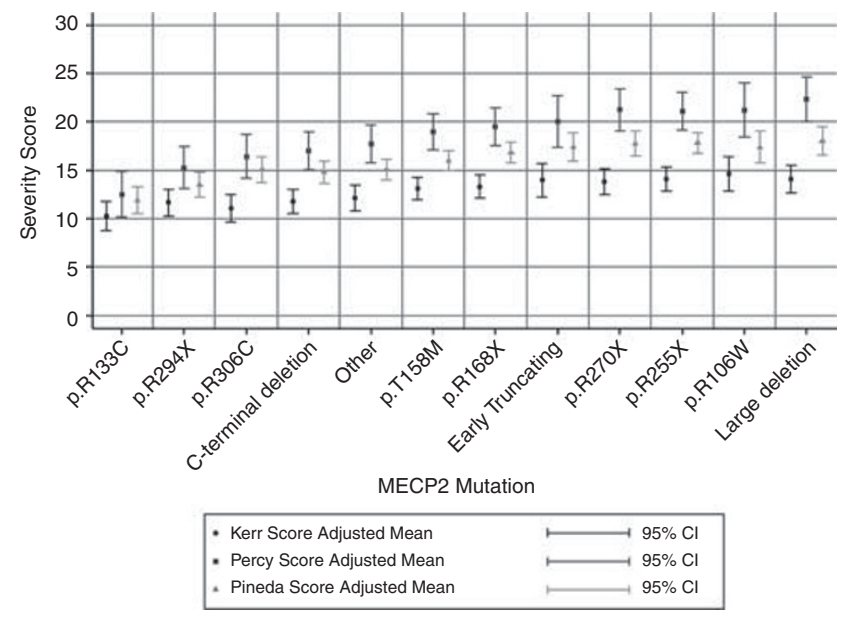

Figure 1 Mean and 95\% confidence interval values for each severity score for each category of common mutation.

Individuals with a large deletion were at higher odds of being in the most severe category for epilepsy (OR 2.72, 95\% CI: 1.38-5.37, $P=0.004$ ), breathing abnormalities (OR 2.19, 95\% CI: $1.17-4.10$, $P=0.015$ ), gross motor function (OR 1.84, 95\% CI: $0.98-3.48$, $P=0.057)$ and scoliosis (OR 1.81, 95\% CI: 0.93-3.49, $P=0.081$ ) after correcting for age and cohort effects. The severity of sleep disturbances (OR 0.90, 95\% CI: 0.48-1.71, $P=0.767$ ) and presence of air swallowing (OR 0.96, 95\% CI: $0.49-1.87, P=0.909$ ) were similar between the two groups (Figure 2).

Using time-to-event (survival) analysis, those with large deletions had an earlier age of onset of scoliosis, seizures and abnormal breathing patterns (Figure 3). Individuals with a large deletion had a median age to development of scoliosis of 6.97 years, compared with 9.44 years for those with the other MECP2 mutations. After adjusting for cohort effects they were at an increased risk of developing scoliosis (HR 2.03, 95\% CI: 1.29-3.22, $P=0.002$ ) compared with those without large deletions. Their median age at onset of seizures was 4.00 years compared with 5.25 years for those with other mutation types, and after adjusting for cohort effects, they were at an increased risk of developing seizures (HR 1.44, 95\% CI: 1.00-2.08, $P=0.050$ ). The median age of onset of breathing abnormalities for those with a large deletion was 3.3 years, somewhat younger than the age of onset for those with other MECP2 mutation types (4 years), and therefore those with large deletions were at a higher hazard of developing breathing abnormalities (HR 1.38, 95\% CI: 0.87- 2.21, $P=0.171$, adjusted for cohort effects). Slightly less than half (42.5\%) of those with a large deletion had learned to walk at time of ascertainment compared with $65.5 \%$ of those with other MECP2 mutations. While half of the individuals with other MECP2 mutations had learned to walk by 2 years, in contrast, fewer than half of those with large deletions had ever learned to walk (longest follow-up was 22 years). Consequently, those with large deletions were at a reduced hazard of learning to walk (HR $0.54,95 \%$ CI: $0.33-0.87, P=0.012$ ). They also had a slightly earlier onset of hand stereotypies, with a median age of onset for those with a large deletion of 1.9 years compared with 2.0 years for those without a large deletion (HR 1.42, 95\% CI: 1.00-2.02, $P=0.047$.

\section{Large deletion case study 1}

This girl was born at 40 weeks gestation via caesarean section after an uneventful pregnancy. She rarely cried during infancy, sat at about 10 months but never learnt to roll. Developmental delay at 12 months led to specialist consultation. Sudden deterioration in speech and hand function occurred at 15 months followed by onset of hand stereotypies at 17 months. Rett syndrome was diagnosed when she was 19 months of age. Genetic testing showed a deletion spanning exons 3 and 4 of the MECP2 gene. Early problems related to breathing disturbances and abdominal bloating, sleep disturbances with night screaming, teeth grinding and constipation. Scoliosis was diagnosed when she was $2 \frac{1}{2} 2$ years old. It increased progressively and surgery was conducted shortly before her 10th birthday. Epilepsy was diagnosed at the age of $3 \frac{1}{2}$ years. It is currently managed with sodium valproate and lamotrigine although she still has several seizures each week. Her feeding skills declined after the onset of epilepsy and a gastrostomy button was later inserted for management of medications and to supplement oral food intake. Now 10 years old, she wakes every night, and often experiences anxiety and panic, screaming and laughing spells during the day or night. Eye gaze is her main method of communication and she has great pleasure completing activities with eye gaze and using a communication board. She is able to touch but not purposefully grasp objects and has never learned to walk. She attends a special school and participates in a range of therapy, music and swimming activities.

\section{Large deletion case study 2}

This girl was born by normal delivery at 35 weeks gestation. During infancy, she sat at 8 months, rolled at 10 months and spoke a few words at 11 months. Subsequent loss of communication skills occurred at 15 months, loss of hand function skills at 24 months and onset of stereotypies at 37 months. She learned to walk at 41 months and was diagnosed with Rett syndrome at 58 months with 


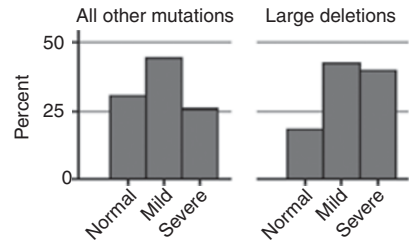

Breathing Abnormalities
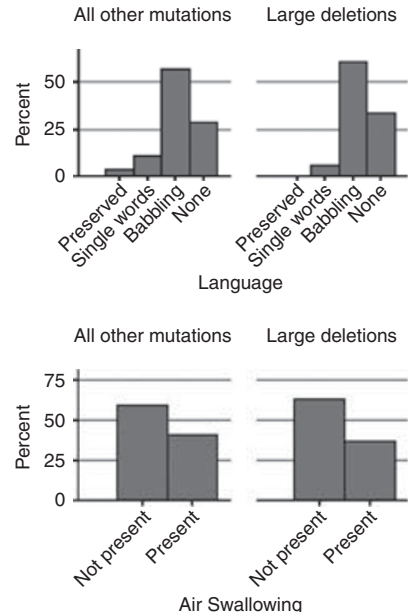
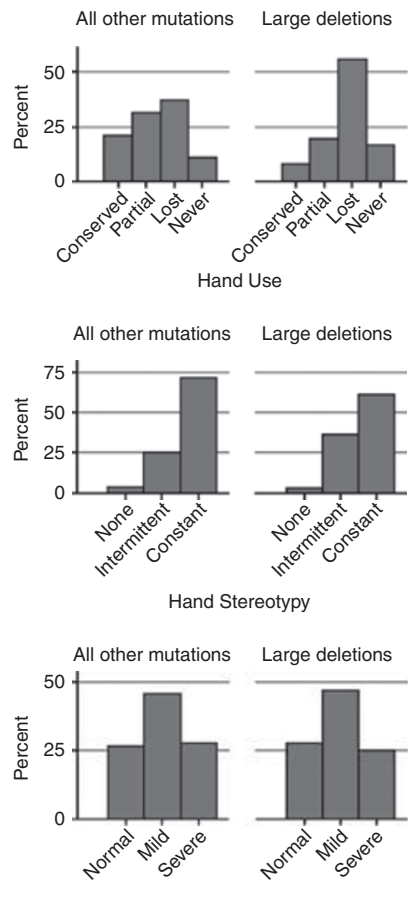

Sleep Disturbance
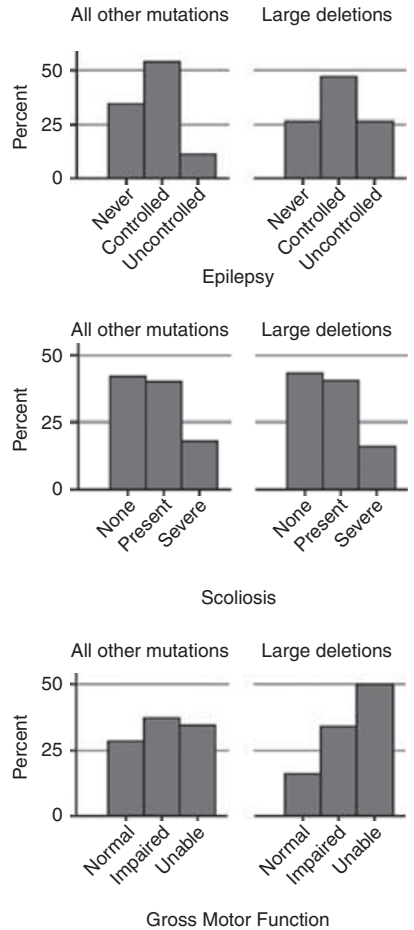

Figure 2 Proportions of individuals with a large deletion or other common mutation for each level of severity in selected items from each of the severity scales. Scoliosis and air swallowing items included data from all individuals; other items did not include Spanish data.

Table 3 Univariate and multivariate results of binary logistic regression analysis of relationships between the presence of a large deletion and aspects of phenotype

\begin{tabular}{|c|c|c|c|c|c|c|}
\hline Factor $^{a}$ & Mutation group (n with data available) & $\mathrm{N}(\%)$ with factor & \multicolumn{2}{|l|}{ Univariate } & \multicolumn{2}{|c|}{ Multivariate ${ }^{\mathrm{b}}$} \\
\hline Ever walked & No large deletion $(n=718)$ & $493(68.7)$ & Baseline & & Baseline & \\
\hline \multirow{2}{*}{ Currently walking } & No large deletion $(n=681)$ & $289(42.4)$ & Baseline & & Baseline & \\
\hline & Large deletion $(n=41)$ & $11(26.8)$ & $0.5(0.24-1.01)$ & 0.05 & $0.53(0.26-1.10)$ & 0.08 \\
\hline Ever talked & No large deletion $(n=687)$ & $344(50.1)$ & Baseline & & Baseline & \\
\hline Ever had hand use & Large deletion $(n=43)$ & $38(88.4)$ & $0.65(0.24-1.72)$ & 0.38 & $0.66(0.24-1.77)$ & 0.40 \\
\hline \multirow[t]{2}{*}{ Maintained hand use } & No large deletion ( $n=632)$ & $165(26.1)$ & Baseline & & Baseline & \\
\hline & Large deletion $(n=38)$ & $6(15.8)$ & $0.53(0.22-1.29)$ & 0.16 & $0.43(0.16-1.10)$ & 0.08 \\
\hline \multirow[t]{2}{*}{ Air swallowing } & No large deletion $(n=643)$ & $270(42.0)$ & Baseline & & Baseline & \\
\hline & Large deletion $(n=42)$ & $15(35.7)$ & $0.77(0.40-1.47)$ & 0.42 & $0.85(0.44-1.65)$ & 0.64 \\
\hline
\end{tabular}

aFor the factor 'currently talking', there were no cases with a large deletion able to talk in comparison with 21 (3.1\%) of all other cases and unable to analyse because of zero frequencies. badjusting for age and data source.

genetic testing indicating a large deletion spanning exons 3 and 4. Epilepsy had previously developed at 32 months and throughout childhood was managed with sodium valproate and lamotrigine, although never completely controlled. Scoliosis developed at $10 \mathrm{l} 1 / 2$ years of age and an anterior spinal fusion was performed at the age of 16 years. She has often hyperventilated and held her breath, as well as experiencing alterations in mood, with day and night screaming and laughing. Now 21 years of age, midazolam is used additionally for seizures, which currently occur approximately monthly. Breathing abnormalities and mood alterations persist. Functional abilities have remained remarkably stable. She utilises eye gaze for communication. She eats more food than would be expected for her size, takes all food and liquids orally and mealtimes take $\sim 30 \mathrm{~min}$. She is able to pick up and hold large and small objects, finger feed, transfer objects from hand to hand and can use a neat pincer grip. Mostly left-handed, she takes keen pleasure in placing wooden puzzles pieces into their correct 

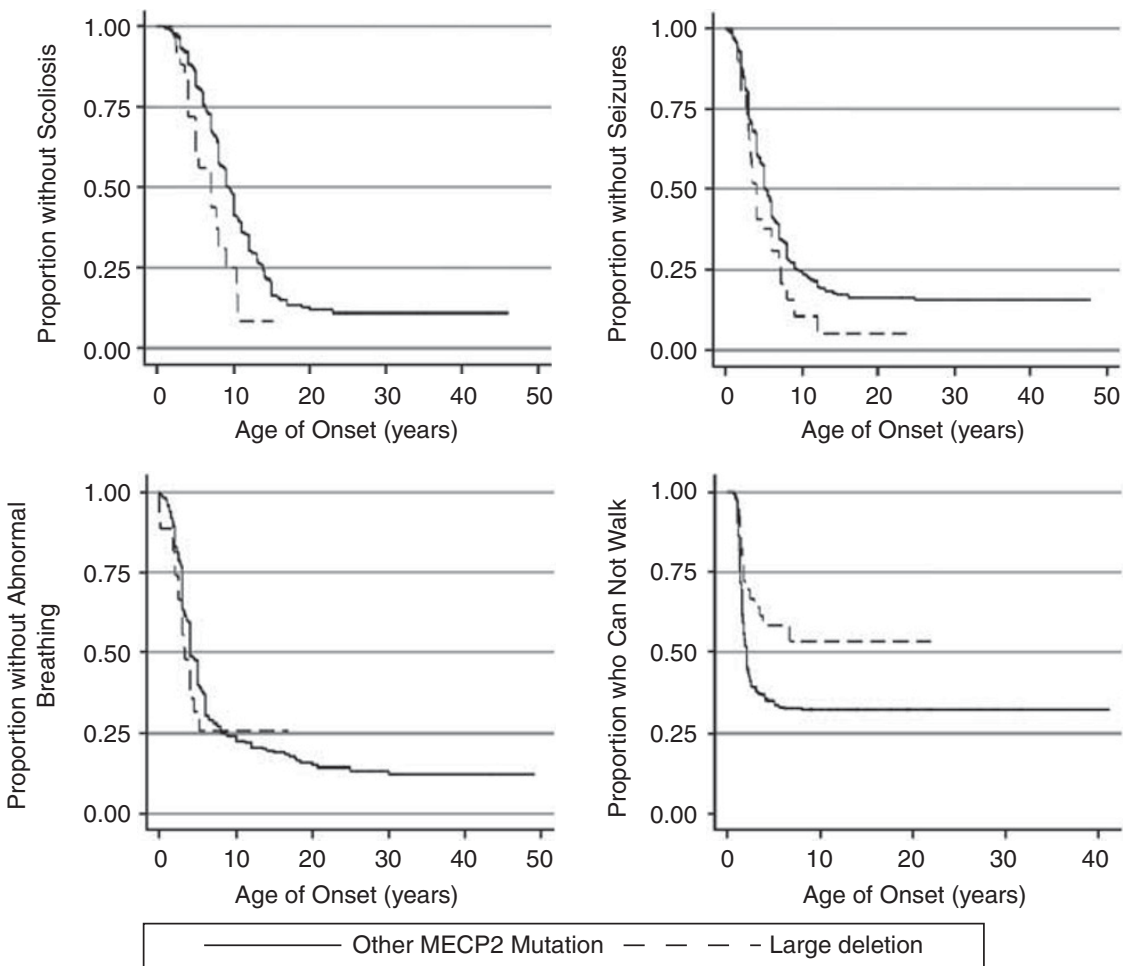

Figure 3 Kaplan-Meier survival estimate of time to age of onset of scoliosis, seizures, ability to walk and abnormal breathing in individuals with and without a large deletion.

position on a board and playing with 'goop'. Late in learning to walk, she walked unsteadily but independently until 12 years of age after which she needed assistance to walk. Now 21 years, she can still walk with light support and negotiate stairs with both hands held, but often chooses to crawl for mobility around the house. She can also walk independently with a walking frame. She previously attended a mainstream school with an assistant and her program included therapies, music hydrotherapy and horse riding. She is currently maintaining participation in regular swimming and home physiotherapy programs.

\section{DISCUSSION}

Using data from the Australian and InterRett databases, we have compared the characteristics of 51 individuals with a large deletion to 923 with other pathogenic MECP2 mutations. We have demonstrated overall that girls and women with a large deletion have the most severe clinical phenotype of the 12 categories of mutations examined. The variation was most marked for the Percy and Pineda scores, which have a greater focus on developmental features than the Kerr score, which relates more to current functioning. The early development of girls with a large deletion was more severely delayed with less likelihood of learning to walk. This severe course continued with greater severity and earlier onset of epilepsy and scoliosis than in those without a large deletion.

Less than half of the girls with a large deletion had learned to walk compared with over two thirds of those with other pathogenic mutations. Moreover, only a quarter of those with a large deletion were still walking compared with $42 \%$ of those with other mutations. We have been able to demonstrate this specific impairment in gross motor function, previously reported in a US study $(n=17$ with large deletion), ${ }^{9}$ both by using logistic regression with ever having walked and currently walking being treated as binary outcomes and in time to event analysis with learning to walk as the outcome. If unable to walk, programs of activities that promote the development and strengthening of abilities to maintain upright postures, such as floor sitting, stool sitting, standing, and assisted walking as able, should be determined on a case-by-case basis. The design of gross motor opportunities for exercise and activities at all levels of function should promote a proactive lifestyle aiming to protect as far as possible against the development of co-morbidities such as scoliosis. ${ }^{22}$

Consistent with the early gross motor impairment, we also noted that a much higher proportion of those with large deletions never developed or completely lost hand function compared with those with other mutations, where a higher proportion had conserved or partially conserved hand function. One characteristic feature of the regression period in Rett syndrome is the development of hand stereotypies. Time to event analysis showed that those with large deletions were likely to have a slightly earlier development of hand stereotypies than those without. In addition to gross motor and hand function the other core feature affected by Rett syndrome is language development. There, we found that a higher proportion of those with large deletions either had no speech or only babbling as opposed to single words or preserved speech compared with those without a large deletion.

Two of the major co-morbidities in Rett syndrome are scoliosis and seizures. We previously found that in the Australian cohort the median age of onset of scoliosis was 9.8 years. ${ }^{23}$ This was a similar age (9.4 years) to what we found in this study for those with other pathogenic mutations. However, for those with a large deletion the median age of onset was much younger at 7.0 years. This might be expected given the high proportion that did not learn to walk and the known association between never walking and an earlier age of scoliosis onset. ${ }^{23,24}$ Similar to scoliosis we found an earlier age of seizure onset in those with large deletions (median age of 48 months) than in those without (median age of 61 months). In a previous Australian study, the median age at 
onset of seizures among the MECP2 mutation groups varied from 37 months in those with a p.R255X mutation to 76 months in cases with the p.R294X mutation and was 42 months for those with a large deletion. ${ }^{25}$ Few studies have examined the relationship between genotype and seizure severity. In another Australian study using data collected in 2000, the seizure rate in those with a C-terminal deletions and in those with a p.R294X mutation was significantly lower than in those with no identified mutation. ${ }^{26}$ In a more recent Italian $^{27}$ but not in a US study, ${ }^{28}$ this result was replicated for C-terminal deletions but not for pR294X mutations. In the 2000 Australian study we were not able to demonstrate an increased seizure rate in those with large deletions, but only five individuals with a large deletion were included. ${ }^{26}$ However, in the present study of 51 individuals, those with a large deletion were at greater odds than those with another pathogenic mutation of having epilepsy of earlier onset and greater severity. Similarly, in the Italian study seizures had occurred in $9 / 10$ of those with large deletions while in the US study ${ }^{28}$ the p.T158M mutation was the mutation where seizures were reported most frequently.

Other morbidities such as sleep problems and breathing abnormalities have been studied even less than seizures in relation to genotype. In a previous Australian study we found that a high prevalence of reported sleep problems and night laughing did appear to be commoner in those with a large deletion. ${ }^{29}$ However, the number of large deletion cases was only five. Although both individuals in the case studies experienced night laughing, we weren't able to systematically examine this in the present study and were unable to replicate our previous findings relating to sleep problems. There is also scant research on relationships between breathing abnormalities and genotype, and this is an area requiring further research.

Although individual studies are showing variation in relation to the effect of genotype on co-morbidities such as seizures, the only other large study that has examined overall severity also found that large deletions had a severe phenotype second only to the mutation p.R168X. ${ }^{9}$ This study also found, as we did, that higher proportions of individuals with large deletions were more impaired in the core domains of ambulation, hand use and language. In the current study, the clinical presentations observed in those with a large deletion were similarly severe to those with the early truncating mutations, including p.R168X, p.R255X and p.R270X. This could relate to greater loss of $\mathrm{MeCP} 2$ including loss of function in the transcriptional repressor domain and nuclear localisation signal areas compared with other mutations, such as p.R133C, p.R306C, p.R294X or the C-terminal deletions, where these areas are less disrupted. Whereas the latter mutations have been associated with milder presentations, earlytruncating mutations have been associated with greater severity. ${ }^{9,10,30}$ Alhough all of the MeCP2 structural domains are necessary to mediate normal cellular actions of $\mathrm{MeCP} 2,{ }^{31}$ it is possible that areas of the central nervous system are differentially susceptible than others to the degree of dysfunction in MeCP2 protein. A less functional protein with greater loss of genetic material could be more disruptive to brainstem and cerebellar structures than a more functional protei$\mathrm{n},{ }^{32}$ and may account for phenotypic differences such as muscle tone and motor function.

Despite the overall severity, as with many of the MECP2 mutation groupings there is clearly considerable variation, which could account for the differing results, for example, in relation to epilepsy. This is the reason why we elected to present two case vignettes, both of which had a large deletion spanning sections of exons 3 and 4 , to demonstrate the contrasting phenotypes, which may result from a large deletion. The first individual is clearly very severe and demonstrates well what might be expected in the phenotype of a large deletion: lack of ambulation, early regression, lack of purposeful hand function and early onset of both seizures and scoliosis. The second case study demonstrates milder symptoms. She learned to walk late, has had and is maintaining hand function at a level $8,{ }^{33}$ is able to crawl and use a Zimmer frame independently and eats well orally. Her functional abilities remain generally strong now she is a young woman and her overall presentation is quite different from the expected phenotype. Despite this she had relatively early onset of seizures and required surgical correction of scoliosis. It was only the large deletion category, which was examined in the US study in relation to X-inactivation, ${ }^{9}$ and it found that there was indeed a relationship between skewing and the ability to walk in these individuals. Such a mechanism could also account for our findings in this young woman.

We acknowledge that factors such as $\mathrm{X}$ inactivation ${ }^{34}$ or BDNF status $^{35}$ could influence the MECP2 mutation, and that this genetic information would be a useful supplement to our analysis. We also recognise that the size of the deletion is variable among individuals and suggest that this be analysed in the future. Although the ARSD is population-based, the InterRett database is not, and the cases ascertained from this database may be subject to selection bias and possibly not fully represent the variability associated with the mutation. On the other hand, in our study the combination of the resources of the two databases has allowed us to accumulate 51 individuals with a large deletion. To our knowledge, this is the largest study to date to describe this mutation and the sample size has allowed use of appropriate statistical methods including survival analysis. Our case studies also use information collected at multiple time points, ${ }^{14}$ and questionnaire data has been supplemented with observed video data of functional abilities that had been collected on two occasions. ${ }^{19,33,36}$ Longitudinal data from different perspectives were able to provide depth to the description of our case studies.

Testing for a large deletion on the MECP2 gene is now widely available and has allowed genetic confirmation of a Rett syndrome diagnosis for such individuals. Our study complements previous smaller studies. For clinicians, we have provided a disorder profile based on the largest dataset of cases with a large deletion; thus, far and although some individuals have milder presentations, the clinical picture is generally severe. This better understanding of the phenotype could assist with counselling and planning of management strategies to address clinical needs. Further research should follow the cohort over time to identify long-term trajectories more clearly.

\section{CONFLICT OF INTEREST}

The authors declare no conflict of interest.

\section{ACKNOWLEDGEMENTS}

The authors would like to acknowledge the International Rett Syndrome Foundation (IRSF previously IRSA) for their ongoing support of the InterRett project and their continued encouragement of this international collaboration. We would also like to express our special appreciation to all the families who have participated in the study and all the clinicians who have completed the questionnaires. In particular, we would like to thank, from the Association Franc, aise du Syndrome de Rett Christiane Roque (past president), Elisabeth Celestin (current president) and Martine Gaudy and Thierry Bienvenu (performed most mutation screening) as well as Yael Yoshei for her assistance to Israeli families. We also acknowledge those members of the InterRett International Reference panel who helped with the piloting of InterRett and the information technology team at the Telethon Institute for Child Health Research for their expertise and assistance. The Australian Rett Syndrome Study was funded by the National Institutes of Health (5R01HD043100-05) and also by the National Health and Medical Research Council (NHMRC) project grant 
303189 for certain clinical aspects. Helen Leonard was previously funded by a NHMRC programme grant 353514 . Her current funding is from an NHMRC Senior Research Fellowship 572568. Alan Percy was supported by NIH grants NIH U54 grant no. HD061222 and IDDRC grant no. HD38985 and the Office of Rare Diseases Research. We would also like to express our sincere gratitude to all the Australian families who have contributed to the study by completing questionnaires, the Australian Paediatric Surveillance Unit (APSU) and the Rett Syndrome Association of Australia who facilitated case ascertainment in Australia. The APSU is a unit of the Division of Paediatrics, Royal Australasian College of Physicians, and is funded by the Department of Health and Ageing and the Faculty of Medicine of the University of Sydney.

1 Neul JL, Kaufmann WE, Glaze DG et al: Rett syndrome: revised diagnostic criteria and nomenclature. Ann Neurol 2010; 68: 944-950.

2 Amir RE, Van den Veyver IB, Wan M et al: Rett syndrome is caused by mutations in X-linked MECP2, encoding methyl- CpG-binding protein 2. Nat Genet 1999; 23: $185-188$.

3 Buyse IM, Fang P, Hoon KT et al: Diagnostic testing for rett syndrome by DHPLC and direct sequencing analysis of the MECP2 gene: identification of several novel mutations and polymorphisms. Am J Hum Genet 2000; 67: 1428-1436.

4 Christodoulou J, Grimm A, Maher T et al: RettBASE: The IRSA MECP2 variation database-a new mutation database in evolution. Hum Mutat 2003; 21: 466-472.

5 Erlandson A, Samuelsson L, Hagberg B et al: Multiplex Ligation-Dependent Probe Amplification (MPLA) Detects Large Deletions in the MECP2 Gene of Swedish Rett Syndrome Patients. Genet Test 2003; 7: 329-332.

6 Archer HL, Whatley SD, Evans JC et al: Gross rearrangements of the MECP2 gene are found in both classical and atypical Rett syndrome patients. J Med Genet 2006; 43: 451-456.

7 Scala E, Longo I, Ottimo $\mathrm{F}$ et al: MECP2 deletions and genotype-phenotype correlation in Rett syndrome. Am J Med Genet A 2007; 143: 2775-2784.

8 Hardwick SA, Reuter K, Williamson SL et al: Delineation of large deletions of the MECP2 gene in Rett syndrome patients, including a familial case with a male proband. Eur J Hum Genet 2007; 15: 1218-1229.

9 Neul JL, Fang P, Barrish J et al: Specific mutations in Methyl-CpG-Binding Protein 2 confer different severity in Rett syndrome. Neurology 2008; 70: 1313-1321.

10 Bebbington A, Percy A, Christodoulou J et al: Updating the profile of C-terminal MECP2 deletions in Rett syndrome. J Med Genet 2010; 47: 242-248.

11 Louise S, Fyfe S, Bebbington A: InterRett, a model for international data collection in a rare genetic disorder. Res Autism Spectr Disord 2009; 3: 639-659.

12 Fehr S, Bebbington A, Nassar N et al: Trends in the diagnosis of Rett syndrome in Australia. Pediatr Res (In press) 2011; 70: 313-319.

13 Laurvick CL, de Klerk N, Bower C et al: Rett syndrome in Australia: a review of the epidemiology. J Pediatr 2006; 148: 347-352.

14 Leonard H, Downs J, Jian L et al: Valproate and risk of fracture in Rett syndrome. Arch Dis Child 2010; 95: 444-448.
15 Fyfe S, Cream A, de Klerk N et al: InterRett and RettBASE: International Rett Syndrome Association databases for Rett syndrome. J Child Neurol 2003; 18: 709-713.

16 Hagberg B, Hanefeld F, Percy A et al: An update on clinically applicable diagnostic criteria in Rett syndrome. Comments to Rett Syndrome Clinical Criteria Consensus Panel Satellite to European Paediatric Neurology Society Meeting, Baden Baden, Germany, 11 September 2001. Eur J Paediatr Neurol 2002; 6: 293-297.

17 Colvin L, Fyfe S, Leonard S et al: Describing the phenotype in Rett syndrome using a population database. Arch Dis Child 2003; 88: 38-43.

18 Young D, Bebbington A, de Klerk $\mathrm{N}$ et al: The relationship between MECP2 mutation type and health status and service use trajectories over time in a Rett syndrome population. Res Autism Spectr Disord 2011; 5: 442-449.

19 Downs J, Bebbington A, Kaufmann WE et al: Longitudinal Hand Function in Rett Syndrome. J Child Neurol 2010; 26: 334-340.

20 Donders AR, van der Heijden GJ, Stijnen T et al: Review: a gentle introduction to imputation of missing values. J Clin Epidemiol 2006; 59: 1087-1091.

21 StataCorp.: Stata Statistical Software. 9th Edition (ed.) Stata Corporation: Texas, 2005.

22 Downs J, Bergman A, Carter P et al: Guidelines for management of scoliosis in Rett syndrome patients based on expert consensus and clinical evidence. Spine 2009; 34: E607-E617.

23 Ager S, Fyfe S, Christodoulou J et al: Predictors of scoliosis in Rett syndrome. J Child Neurol 2006; 21: 809-813.

24 Percy AK, Lee HS, Neul JL et al: Profiling scoliosis in Rett syndrome. Pediatr Res 2010; 67: 435-439.

25 Jian L, Nagarajan L, de Klerk $\mathrm{N}$ et al: Predictors of seizure onset in Rett syndrome. J Pediatr 2006; 149: 542-547.

26 Jian L, Nagarajan L, de Klerk N et al: Seizures in Rett syndrome: An overview from a one-year calendar study. Eur J Paediatr Neurol 2007; 11: 310-317.

27 Pintaudi M, Calevo MG, Vignoli A et al: Epilepsy in Rett syndrome: Clinical and genetic features. Epilepsy Behav 2010; 19: 296-300.

28 Glaze DG, Percy AK, Skinner S et al: Epilepsy and the natural history of Rett syndrome. Neurology 2010; 74: 909-912.

29 Young D, Nagarajan L, de Klerk N et al: Sleep problems in Rett syndrome. Brain Dev 2007; 29: 609-616.

30 Bebbington A, Anderson A, Ravine D et al: Investigating genotype-phenotype relationships in Rett syndrome using an international dataset. Neurology 2008; 70: 868-875.

31 Hite KC, Adams VH, Hansen JC: Recent advances in MeCP2 structure and function. Biochem Cell Biol 2009; 87: 219-227.

32 Shahbazian MD, Zoghbi HY: Rett Syndrome and MeCP2: Linking Epigenetics and Neuronal Function. Am J Hum Genet 2002; 71: 1259-1272.

33 Downs J, Bebbington A, Jacoby $\mathrm{P}$ et al: Level of purposeful hand function as a marker of clinical severity in Rett syndrome. Dev Med Child Neurol 2010; 52: 817-823.

34 Archer $\mathrm{H}$, Evans J, Leonard $\mathrm{H}$ et al: Correlation between clinical severity in patients with Rett syndrome with a p.R168X or p.T158M MECP2 mutation, and the direction and degree of skewing of X-chromosome inactivation. J Med Genet 2007; 44: 148-152.

35 Ben Zeev B, Bebbington A, Ho G et al: The common BDNF polymorphism may be a modifier of disease severity in Rett syndrome. Neurology 2009; 72: 1242-1247.

36 Foley K, Downs J, Bebbington A et al: Change in gross motor abilities of girls and women with Rett syndrome over a three to four year period. J Child Neurol In press 2011; 26: 1237-1245. 